\title{
Mild COVID-19 infection in a group of teriflunomide-treated patients with multiple sclerosis
}

\author{
Vittorio Mantero ${ }^{1}$ (D) Damiano Baroncini ${ }^{2} \cdot$ Roberto Balgera $^{1} \cdot$ Clara Guaschino $^{2} \cdot$ Paola Basilico $^{1} \cdot$ Pietro Annovazzi $^{2}$. \\ Mauro Zaffaroni ${ }^{2} \cdot$ Andrea Salmaggi $^{1} \cdot$ Christian Cordano $^{3}$
}

Received: 16 June 2020 / Revised: 20 August 2020 / Accepted: 27 August 2020 / Published online: 31 August 2020

c) Springer-Verlag GmbH Germany, part of Springer Nature 2020

\section{Dear Editor,}

We have read with interest the recent paper published in this journal by Maghzi et al. [1] reporting a case series of five teriflunomide-treated multiple sclerosis (MS) patients who developed active COVID-19 infection and continued their therapy with a self-limiting infection, without any relapse. The authors hypothesized that, in these cases, COVID19 infection could have had a better outcome because of the immune-biologic mechanisms pertaining to teriflunomide. In line with this hypothesis, Möhn and colleagues [2] reported another case of a 42-year-old male MS patient that, despite treatment with teriflunomide and high-dose methylprednisolone pulse therapy, developed a mild course of COVID-19.

To date, no other cases of COVID-19 teriflunomidetreated patients were reported.

We present here a case series of six patients treated with teriflunomide that developed a self-limiting COVID-19 infection. Diagnosis was confirmed in three of them with PCR from nasopharyngeal swabs and/or serology, while in the other three patients the diagnosis was based on typical symptomatology after a contact with COVID-19 patients during the epidemic peak in Lombardy between March and May 2020. All patients continued their therapy, and none of them experienced an MS relapse during the COVID19 symptoms. Clinical characteristics and hematological

Vittorio Mantero

vittorio.mantero@hotmail.com

1 Department of Neurology, MS Center, ASST Lecco, Via dell'Eremo 9/11, 23900 Lecco, Italy

2 MS Center, Gallarate Hospital, ASST Valle Olona, Gallarate, Italy

3 Department of Neurology, UCSF Weill Institute for Neurosciences, University of California, San Francisco, San Francisco, CA, USA values are reported in table in Table 1. Patients were mostly female $(67 \%)$, with an average age of $45( \pm 8.6)$ years and a disease duration of $15.8( \pm 9.6)$ years. Median EDSS was 1.75 (range 1-4.5), and the average time on treatment with teriflunomide was $2.1( \pm 1.6)$ years. None of these patients presented relevant comorbidities.

None had lymphopenia, neutropenia or leukopenia. No patient required hospitalization, ICU care, or intubation. They all improved without receiving any specific treatment. One of the patients reported subjective diplopia, interpreted as a pseudo-relapse by the treating neurologist. Compared with the patients presented by Maghzi et al., the patients in our cohort were 8.6 years younger, average time on treatment with teriflunomide was about 0.5 years shorter, disease duration was 3 years longer and median EDSS was 0.75 point lower (2.5 with range 0-6 in Maghzi et al.). A weakness of our series is that infection was confirmed only in three patients.

Generally, patients with MS have an increased risk of infections [3], with about twice the risk of hospitalization with respect to the general population [4]. Infections also have a role in triggering MS relapses or worsening pre-existing MS symptoms [5], and they are more likely to happen in patients treated with disease-modifying therapies (DMTs), with a different risk depending on the mechanism of action of the drug [3].

The risk and course of COVID-19 in patients with MS is still unclear. Sormani et al. reported on 232 patients with MS from 38 different Italian MS centers presenting COVID-19 infection, 223 of whom experienced mild symptoms, 4 experienced severe infection and 6 with critical conditions [6]. In a study by Safavi et al., patients who were on a B-cell depleting antibody were at a higher risk of developing COVID-19 infection and being hospitalized [7]. Few reports about other therapies have been reported in the literature [8-12].

Teriflunomide is the active metabolite of leflunomide. Cai et al. reported a case of a patient with rheumatoid arthritis 
Table 1 Summary of cases

\begin{tabular}{llllllllll}
\hline Patient & Age & Sex & MS type & $\begin{array}{l}\text { MS } \\
\text { duration } \\
\text { (years) }\end{array}$ & $\begin{array}{l}\text { EDSS } \\
\text { Years on } \\
\text { terifluno- } \\
\text { mide }\end{array}$ & ALC* & CBC/LFTs* & Comorbidities \\
\hline 1 & 37 & F & RR & 19 & 1.5 & 0.5 & WNL & WNL & No \\
2 & 54 & F & RR & 32 & 3.5 & 1 & WNL & WNL & No \\
3 & 57 & F & RR & 22 & 1 & 0.5 & WNL & WNL & No \\
4 & 40 & M & RR & 3 & 1 & 3 & WNL & WNL & No \\
5 & 48 & M & RR & 10 & 2 & 2.5 & WNL & WNL & No \\
6 & 34 & F & RR & 9 & 4.5 & 5 & WNL & WNL & No \\
\hline
\end{tabular}

$A L C$ absolute lymphocyte count, $C B C$ complete blood count, EDSS Expanded Disability Status Scale score, $L F T$ s liver function tests, $M S$ multiple sclerosis, $R R$ relapsing-remitting, $W N L$ within normal limits

*At nearest available time preceding COVID-19 infection

treated with leflunomide presenting a COVID-19 infection complicated by severe symptoms [13].

Our small case series, although in a different population compared to the paper of Maghzi and coauthors [1], seem to confirm that continuing therapy might be safe in teriflunomide-treated MS patients who develop COVID-19 infection. Withdrawal of teriflunomide does not seem to be necessary, especially if the lymphocyte count is higher than $500-800 / \mathrm{mm}^{3}$ [14].

Author contributions VM contributed to the conceptualization, gathering of data and drafting the manuscript; DB, RB, CG, PB, PA contributed to gathering of data and drafting the manuscript; $\mathrm{MZ}, \mathrm{AS}$, and $\mathrm{CC}$ contributed to drafting and revising the manuscript.

\section{Compliance with ethical standards}

Conflicts of interest VM, DB, RB, CG, PB, PA and MZ served in advisory boards and/or received travel grant from Genzyme for participation at congress. AS and $\mathrm{CC}$ have no conflict of interest to disclose.

Ethical approval All procedures performed in this study were in accordance with the ethical standards of the institutional and national research committee and with the 1964 Helsinki declaration and its later amendments or comparable ethical standards.

Informed consent All patients provided consent to be anonymously included in this report.

\section{References}

1. Maghzi AH, Houtchens MK, Preziosa P, Ionete C, Beretich BD, Stankiewicz JM, Tauhid S, Cabot A, Berriosmorales I, Schwartz THW, Sloane JA, Freedman MS, Filippi M, Weiner HL, Bakshi R (2020) COVID-19 in teriflunomide-treated patients with multiple sclerosis. J Neurol 3:1-7. https://doi.org/10.1007/s0041 5-020-09944-8

2. Möhn N, Saker F, Bonda V et al (2020) Mild COVID-19 symptoms despite treatment with teriflunomide and high-dose methylprednisolone due to multiple sclerosis relapse. J Neurol 28:1-3. https://doi.org/10.1007/s00415-020-09921-1
3. Luna G, Alping P, Burman J et al (2020) Infection risks among patients with multiple sclerosis treated with fingolimod, natalizumab, rituximab, and injectable therapies. JAMA Neurol 77:184-191. https://doi.org/10.1001/jamaneurol.2019.3365

4. Wijnands JM, Kingwell E, Zhu F, Zhao Y, Fisk JD, Evans C, Marrie RA, Tremlett $H$ (2017) Infection-related health care utilization among people with and without multiple sclerosis. Mult Scler 23(11):1506-1516

5. Steelman AJ (2015) Infection as an environmental trigger of multiple sclerosis disease exacerbation. Front Immunol 6:520. https ://doi.org/10.3389/fimmu.2015.00520

6. Sormani MP (2020) An Italian programme for COVID-19 infection in multiple sclerosis. Lancet Neurol. https://doi.org/10.1016/ S1474-4422(20)30147-2

7. Safavi F, Nourbakhsh B, Azimi AR (2020) B-cell depleting therapies may affect susceptibility to acute respiratory illness among patients with multiple sclerosis during the early COVID-19 epidemic in Iran. Mult Scler Relat Disord. https://doi.org/10.1016/j. msard.2020.102180

8. Foerch C, Friedauer L, Bauer B, Wolf T, Adam EH (2020) Severe COVID-19 infection in a patient with multiple sclerosis treated with fingolimod. Mult Scler Relat Disord 42:102180. https://doi. org/10.1016/j.msard.2020.102180

9. Novi G, Mikulska M, Briano F et al (2020) COVID-19 in a MS patient treated with ocrelizumab: does immunosuppression have a protective role? Mult Scler Relat Disord 42:102120. https://doi. org/10.1016/j.msard.2020.102120

10. Carandini T, Pietroboni AM, Sacchi L et al (2020) Alemtuzumab in multiple sclerosis during the COVID-19 pandemic: a mild uncomplicated infection despite intense immunosuppression. Mult Scler. https://doi.org/10.1177/1352458520926459

11. Aguirre C, Meca-Lallana V, Barrios-Blandino A, Del Río B, Vivancos J (2020) Covid-19 in a patient with multiple sclerosis treated with natalizumab: may the blockade of integrins have a protective role? Mult Scler Relat Disord 44:102250. https://doi. org/10.1016/j.msard.2020.102250

12. Mantero V, Abate L, Basilico P, Balgera R, Salmaggi A, Nourbakhsh B, Cordano C (2020) COVID-19 in dimethyl fumaratetreated patients with multiple sclerosis. J Neurol 25:1-3. https:// doi.org/10.1007/s00415-020-10015-1

13. Cai S, Sun W, Li M, Dong L (2020) A complex COVID-19 case with rheumatoid arthritis treated with tocilizumab. Clin Rheumatol 39:2797-2802. https://doi.org/10.1007/s10067-020-05234-w

14. Brownlee W, Bourdette D, Broadley S, Killestein J, Ciccarelli O (2020) Treating multiple sclerosis and neuromyelitis optica spectrum disorder during the COVID-19 pandemic. Neurology 94:949-952. https://doi.org/10.1212/WNL.0000000000009507 